\title{
Evaluating Published Skin Dose Tolerance Limits for Stereotactic Body Radiation Therapy of Lung Cancer
}

Jimm Grimm ${ }^{1}$, Tamara LaCouture ${ }^{2}$, Sucha O. Asbell ${ }^{2}$, Noel Kramer ${ }^{2}$, Niraj Pahlajani ${ }^{2}$, Nazish Ahmad ${ }^{2}$, Roma Srivastava ${ }^{2}$, Yan Chen ${ }^{2}$, Qianyi Xu ${ }^{2}$, Raymond Croce ${ }^{2}$, Meng Chew ${ }^{3}$ , Jinyu Xue ${ }^{2}$

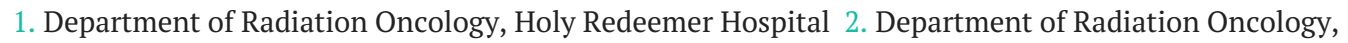
Cooper University Hospital 3. Department of Mechanical Engineering, Lehigh University, Bethlehem, PA

$\square$ Corresponding author: Jimm Grimm, jimmgrimmjr@yahoo.com Disclosures can be found in Additional Information at the end of the article

\section{Abstract}

Objectives: Published local control rates for certain stereotactic body radiotherapy (SBRT) lung cancer treatments have exceeded 97\%, but several serious adverse events have been reported. No five-year normal tissue complication probability (NTCP) statistical analysis SBRT results have been published yet for skin. While we expectantly await these publications, the goal of this manuscript is to glean the published expert opinion and ongoing clinical trials for skin dose tolerance limits in one to five fractions for clinical application and research.

Methods: All 42 lung tumors treated with the CyberKnife at Cooper University Hospital from July 2008 to May 2010 that had skin contours and Monte Carlo dose calculations with heterogeneity corrections were reviewed for toxicity. Twenty-one published dose tolerance limits for skin were partitioned into high-risk and low-risk categories. The DVH Evaluator software tool was developed by the first author and used to conveniently assess the dose tolerance limits for each case prior to treatment and to analyze the followup data.

Results: Three of the 42 patients experienced mild Grade 1 erythema that resolved within a few months. No Grade 2 or higher adverse events of skin toxicity were encountered. A unified framework of high-risk and low-risk skin dose limits in one to five fractions at specific dosevolume levels is presented.

Conclusions: When the dose tolerance limits are diligently studied and respected, SBRT has potential to be a safe and effective form of radiation therapy. Longer follow-up will still be required to statistically analyze late effects and long-term outcomes.

Review began 06/04/2012 Published 06/27/2012

\section{Copyright 2012}

Grimm et al. This is an open access article distributed under the terms of the Creative Commons Attribution License CC-BY 3.0., which permits unrestricted use, distribution, and reproduction in any medium, provided the original author and source are credited.
Categories: Radiation Oncology

Keywords: cyberknife, erythema, skin, lung cancer, dose tolerance limits, adverse events, stereotactic body radiation therapy, dvh evaluation

\section{Introduction}

Published three-year local control rates for stereotactic body radiation therapy (SBRT) for primary lung cancer have exceeded $97 \%$ [1] and the potential benefit to patients is enormous. However, adverse events (AE) as high as Grade 5 (i.e., death) have also resulted from SBRT [2, 3], so extreme caution must be employed. At Cooper University Hospital (CUH), the published 
SBRT dose tolerance limits have been diligently studied and respected and the occurrence of Grade 3 (G3) or higher adverse events from treatment has been extremely rare in our clinic.

Most current publications that present SBRT dose tolerance limits unfortunately do not state the estimated associated risk level. For conventional fractionation schemes, Emami's presentation of the $5 \%$ and $50 \%$ tolerance dose (TD) for adverse event in five years (TD $5 / 5$ and TD 50/5) has been extremely useful [4]. The recent QUANTEC publications have also presented statistically estimated dose tolerance limits for many critical structures [5]. Physicians can assess the doses to critical structures with respect to the TD 5/5 and TD 50/5 reference levels to ensure appropriate risk for the staging and condition of each patient. However, for the SBRT dose tolerance limits that have been presented to date, such classification has generally not been made, and unfortunately, QUANTEC did not include skin dose tolerance limits for SBRT. This makes it quite a challenge to ensure that the risk level of each SBRT treatment is appropriate for each patient.

Many important publications have already presented skin dose tolerance limits [6-10]. Numerous excellent SBRT protocols, including skin dose tolerance limits, also have been completed and others are still underway [11-15]. Ultimately, we will want to know the normal tissue complication probability (NTCP) statistically analyzed TD 5/5 and TD 50/5 tolerance doses for SBRT, but no five-year SBRT dose tolerance NTCP results have been published yet for skin. In the meantime, we propose classification of SBRT dose tolerance limits into "low-risk" and "high-risk" categories. As long-term follow-up data emerges, the low-risk limits will evolve towards the TD 5/5, and the high-risk limits will evolve towards the TD 50/5. At the present time, we do not have sufficient data to numerically estimate the risk levels for most critical structures, but we do have enough dose tolerance limits to begin the classification.

Skin dose can be a limiting factor in SBRT, several adverse events have been reported [7, 9-10, $16,17]$, and we have accumulated sufficient information to share some observations. For these reasons, from the CUH CyberKnife patient data and an extensive literature review, we will use skin dose tolerance as an example to establish a set of reliable low-risk limits, and to formulate a unified framework into which SBRT dose tolerance limits can be expressed.

\section{Materials And Methods}

Among the 234 cases that were treated with the CyberKnife (Accuray, Inc., Sunnyvale, Ca, USA) at Cooper University Hospital from July 2008 to May 2010, there were 42 lung cancer cases that had skin contours and Monte Carlo dose calculations with heterogeneity corrections. All 42 of these cases were evaluated in this retrospective study (IRB \#10-094EX) for skin toxicity. For this study, we define skin as a $5 \mathrm{~mm}$ thick shell from the patient's surface to $5 \mathrm{~mm}$ deep.

Patient ages ranged from 37 to 88 years of age. Tumor sites were not restricted from any area of the lung. Patients were treated primarily for either early stage (T1 - T2NOM0) lung cancer or for metastatic lung nodules, without previous radiation exposure in the treatment area.

Prescribed doses ranged from $28.5 \mathrm{~Gy}$ to $60 \mathrm{~Gy}$ in three to five fractions, delivered in three to 18 days (two to 17 elapsed days). Only three patients had 11 elapsed days or more. The physicians carefully assessed the risk benefit ratio for each patient, using $60 \mathrm{~Gy}$ in three fractions most of the time but using a lower dose whenever needed, as low as 28.5 Gy in three fractions or 32.5 Gy in five fractions for some tumors near the mediastinum. If a patient were to receive chemotherapy, it was held for two weeks prior to and after SBRT. Skin doses ranged from negligible to 40Gy in three to five fractions.

The primary endpoint that the published dose tolerance limits for skin relate to is Grade 3 radiation dermatitis or ulceration unless otherwise noted. Some publications also mention 
Grade 3 fibrosis requiring intervention [17] or Grade 4 necrosis [16] as possible endpoints associated with their skin dose tolerance limits.

All CUH patient doses reported in this manuscript have been calculated using Accuray's Monte Carlo dose calculation algorithm with heterogeneity corrections. The Dose Volume Histogram (DVH) of each patient was evaluated with respect to specified dose tolerance limits prior to treatment as seen in the example in Fig. 1, using the DVH Evaluator software tool which is an FDA-cleared product that is distributed by LifeLine Software (Austin, Tx) and was developed by the first author (information is available at www.DiversiLabs.com). All subsequent adverse events according to the CTCAE v3 definitions [18] were recorded and analyzed using the DVH Evaluator. Explicit quantitative data can be displayed for each patient; as in Fig. 1, it can clearly be seen that for the sample patient the three-fraction RTOG 0618 Total Lung V20Gy $\leqslant$ $10 \%$ limit has been exceeded but the minor deviation limit of V20Gy $\leqslant 15 \%$ has been met. The 24 Gy maximum skin dose limit of the protocol has been exceeded by a large amount as will be discussed in the results. The left bronchus exceeds the $30 \mathrm{~Gy}$ max dose limit, but less than $1 / 100^{\text {th }}$ of one cubic centimeter (cc) of volume actually is in excess. All other critical structures meet the protocol limits so they have not been plotted.

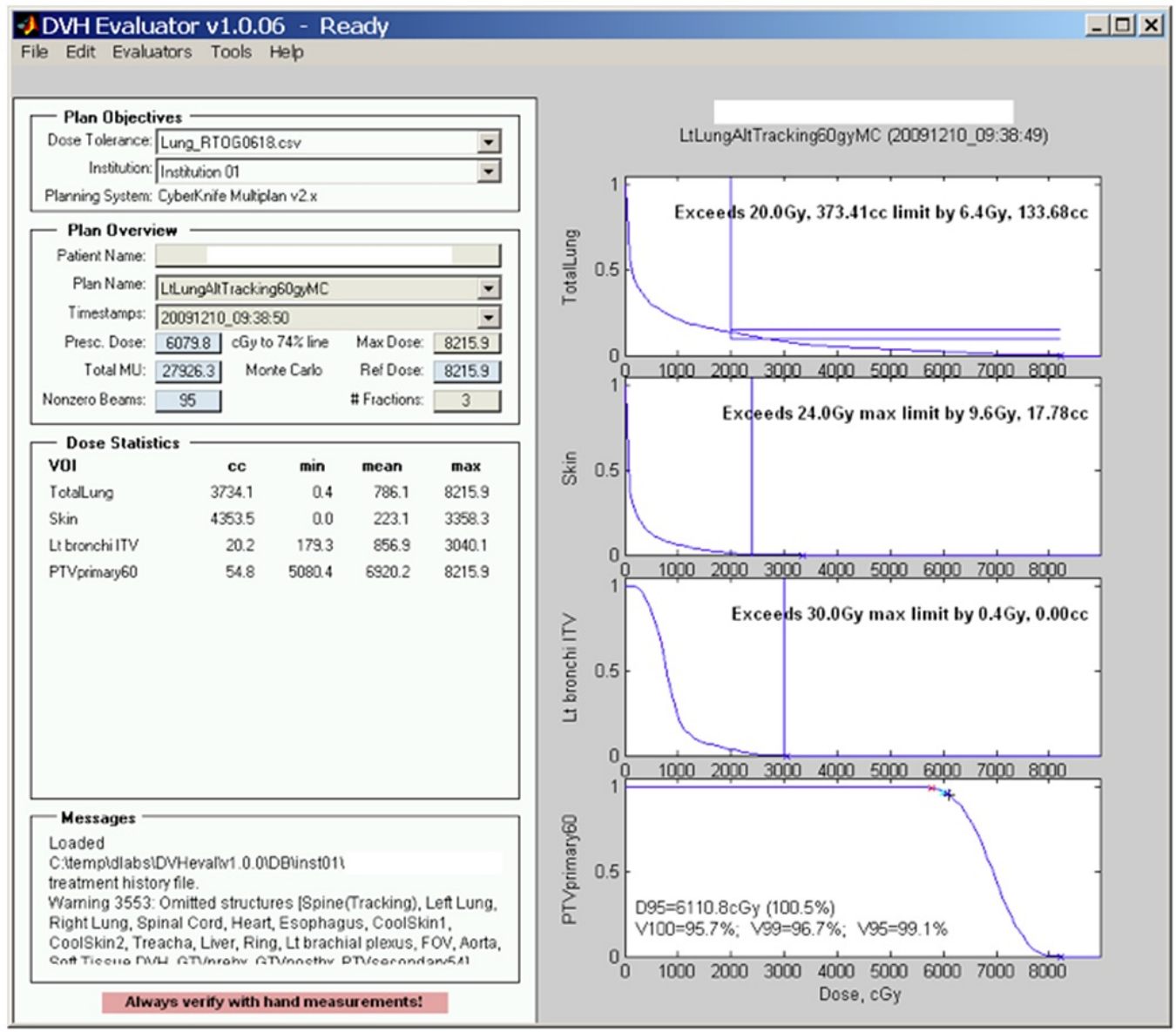

FIGURE 1: RTOG 0618 dose tolerance limits superimposed on a sample patient's DVH plots in the DVH Evaluator software tool.

RTOG 0618 dose tolerance limits superimposed on a sample patient's DVH plots in the DVH Evaluator software tool. The curved lines represent the patient's DVH for each anatomical structure; the horizontal and vertical lines represent the volume and dose of the tolerance limits specified by the RTOG 0618 protocol, respectively; and the space between the DVH and the 


\section{Results}

This study superimposes two types of results: published dose tolerance limits and clinical patient data. We performed an extensive literature review and found more than 500 published SBRT dose tolerance limits for anatomical critical structures throughout the body in one to five fractions [19]. Twenty one dose tolerance limits were for skin, and those are summarized in Table 1 and are shown in the DVH Risk Map in Fig. 2. The first column in Table 1 shows the number of fractions for each dose tolerance limit. For dose-volume limits, columns two and three show the specified volume and corresponding allowable dose, respectively. For max dose limits, column four has the allowable maximum dose. Columns three and four are mutually exclusive; only one type of dose tolerance limit is allowed per row. If a publication presented adverse event statistics, column five has the number of patients for whom the adverse event occurred and column seven has the number of patients in the study. In column six, it can be seen that unfortunately only one of the references actually reported the number of patients who exceeded the specified dose tolerance limit, although this quantity is among the most important information. Column eight provides all references we found that mention the dose limit, and some brief notes are occasionally mentioned in the last column. For simplicity in this manuscript, all doses are plotted on a linear scale and all interpolations are linear; no conversions for biological effective dose (BED) have been applied. 


\section{Cureus}

\begin{tabular}{|c|c|c|c|c|c|c|c|c|}
\hline & & & & \# & \# Pts & & & \\
\hline & & cc & $\operatorname{Max}$ & $\mathrm{AE}$ & $\mathrm{Rx}$ & \# Pts & & \\
\hline \# & Volume & Limit & Limit & $\geq$ & This & in & & \\
\hline$F x$ & cc & Gy & Gy & G3 & Dose & Study & Refs. & Notes \\
\hline 1 & 0.035 & 26 & & & & & 12 & RTOG 0631 \\
\hline 1 & & & 26 & & & & 14 & RTOG 0915 \\
\hline 1 & & & 16 & & & & 6 & \\
\hline 1 & 10 & 23 & & & & & 12,14 & RTOG 0631\&0915 \\
\hline 1 & 10 & 14.4 & & & & & 6 & \\
\hline 3 & & & 54 & 3 & & 50 & 16 & G4 necrosis \\
\hline 3 & & & 48 & 1 & & 21 & 17 & 48 Gy $1 \mathrm{~cm}$ deep, G3 fibrosis \\
\hline 3 & 1 & 35 & & & & & 15 & Accuray STARS \\
\hline 3 & 10 & 30 & & & & & 15 & Accuray STARS \\
\hline 3 & & & 30 & 1 & & 38 & 7 & 30 Gy $1 \mathrm{~mm}$ from surface \\
\hline 3 & & & 24 & & & & 6,11 & RTOG 0618 \\
\hline 3 & & & 21 & & & & 7 & \\
\hline 3 & 10 & 22.5 & & & & & 6 & \\
\hline 3 & & & 20 & & & & 8 & \\
\hline 4 & 1 & 40 & & & & & 9,15 & Accuray STARS, Surface to $5 \mathrm{~mm}$ \\
\hline 4 & 10 & 35 & & 3 & & 27 & 9,15 & Accuray STARS, Surface to $5 \mathrm{~mm}$ \\
\hline 4 & & & 36 & & & & 14 & RTOG 0915 \\
\hline 4 & 10 & 33.2 & & & & & 14 & RTOG 0915 \\
\hline 4 & 50 & 30 & & 5 & 67 & 268 & 10 & G2 skin toxicity \\
\hline 5 & & & 32 & & & & 6,13 & RTOG 0813, QOD \\
\hline 5 & 10 & 30 & & & & & 6,13 & RTOG 0813, QOD \\
\hline
\end{tabular}

TABLE 1: Published SBRT dose tolerance limits for skin

The information in Table 1 is extremely important, but when these limits are applied clinically, it is apparent that many limits are missing or conflict with one another. For example, we found no published skin dose limits for two fractions, and the volumes specified by the different 
authors are widely varying, from $0.035 \mathrm{cc}$ up to 50cc. The Accuray STARS protocol [15] allows $1 \mathrm{cc}$ to receive $40 \mathrm{~Gy}$ in four fractions, whereas RTOG 0813 only allows $32 \mathrm{~Gy}$ to the maximum in five fractions. How can all these sparse and discordant data be combined into unified SBRT dose tolerance limits for clinical application? The DVH Risk Map in Fig. 2 organizes all the available information into a unified format without gaps. The initial values are preliminary based upon the expert opinion limits in Table 1, and as the long term statistical information emerges, the data points can be replaced one by one with the true TD 5/5 and TD 50/5 values. Each set of unified SBRT dose tolerance limits includes five values: two percentage volume limits are given, as well as two absolute volume limits and a maximum point dose limit. Corresponding to these five values, the DVH Risk Map in Fig. 2 has five subplots, and in the tabular format in the bottom of the figure, the low-risk and high-risk sections both have five columns.

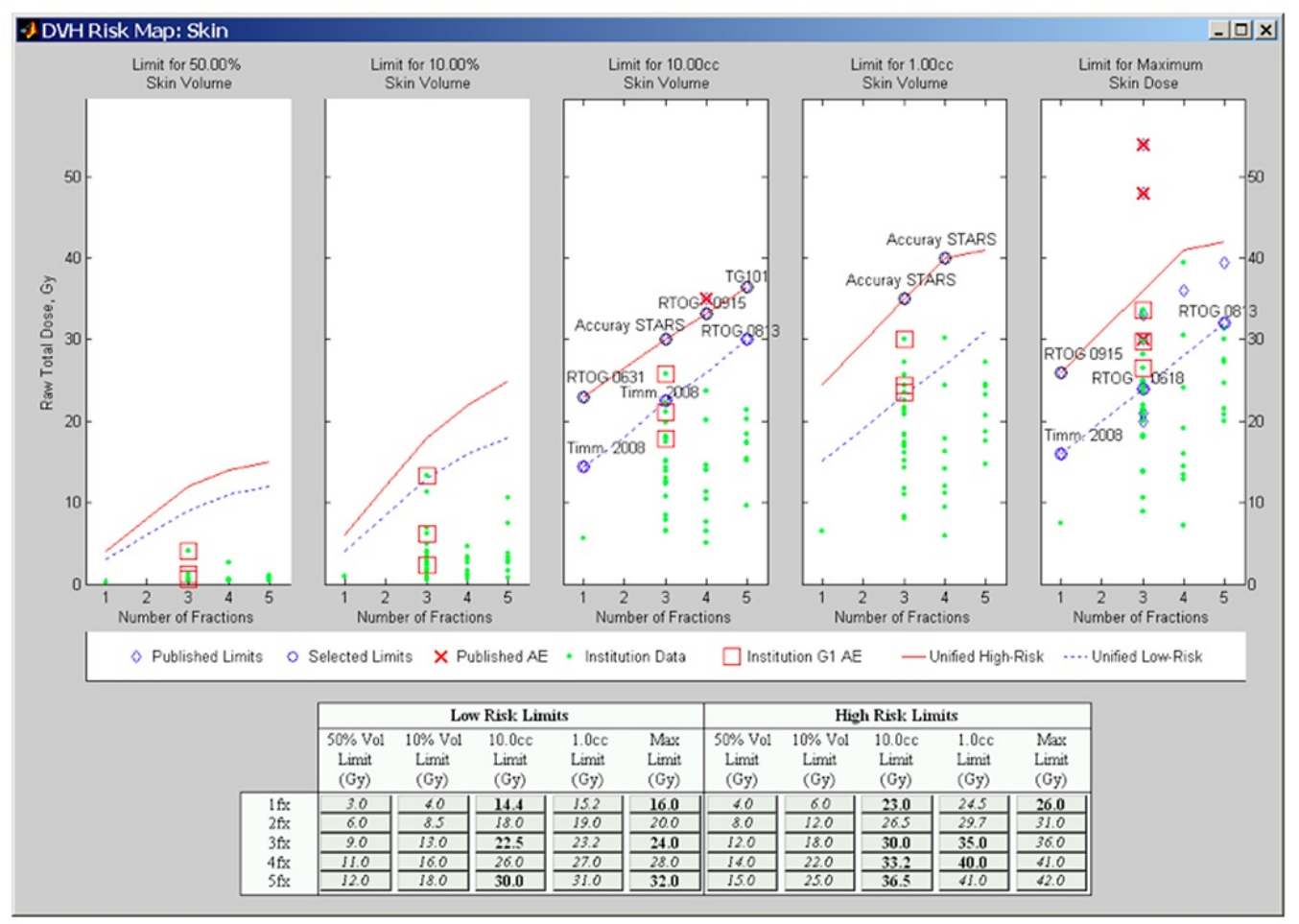

\section{FIGURE 2: DVH Risk Map including published SBRT dose tolerance limits for skin superimposed on CUH patient data}

DVH Risk Map including published SBRT dose tolerance limits for skin superimposed on CUH patient data. (Bolded limits are published data and italicized are interpolated or extrapolated.)

In conventional radiation therapy the fractional doses are low enough that the patient's body can repair sublethal damage to healthy tissue in time for the next fraction, while slowly killing the tumor. In contrast, the goal of SBRT is to overwhelm the tumor with an ablative dose. As shown in Table 1, in many cases small volumes of healthy tissue can still tolerate these higher doses per fraction. Implicit in this rationale is the requirement that the cumulative dose to large volumes of healthy tissue must still remain at the biological equivalent of conventional tolerance doses. Neglecting this implicit constraint could result in ablation of large volumes of critical structures. We prefer to make this large-volume requirement explicit so the normal tissue effects can be studied quantitatively. For these reasons, the leftmost two unified dose tolerance limits in each set are percentage volume limits. For most critical structures in SBRT, 
the most important dose tolerance limits will be to a small absolute volume, but the percentage volume limits can provide additional information. We use the autocontour feature of the planning system to contour the entire structure, so the percentage volume skin limits include all the skin in the CT scan.

Several authors have already specified SBRT dose tolerance limits for various anatomical critical structures in terms of the $50 \%$ volume (19) and it is conveniently midway between Emami's tolerance limits for $1 / 3$ and 2/3 volumes, so in our institution we use it for every critical structure throughout the body. The dose to $50 \%$ of the skin in the entire CT scan should always be low for SBRT. If it is much higher than 3 Gy per fraction, one might question if this SBRT plan is the best treatment option, because this might indicate a very large target. This dose limit therefore could be helpful in gauging the consequences of treating a large tumor with SBRT. We typically scan $15 \mathrm{~cm}$ inferior and superior to the tumor, and the total skin volume can vary from 1500 to 3000cc or more, so even with fairly consistent CT scan length there is still quite a bit of variation in total skin volume due to body habitus. Despite this variation, it may be worth noting from Fig. 2 that the three patients who experienced side-effects had among the highest doses to large percentage volumes as well, so it is likely that future studies could quantify correlations in the percentage volume limits.

The $10 \%$ volume has been chosen for evaluation because it is smaller than Emami's previously described $1 / 3$ volume, since the volumes relevant to SBRT are typically smaller than the relevant volumes for conventional radiotherapy. A small percentage volume limit is necessary because the maximum, 1cc, or 10cc limits do not indicate the size of the high dose region. Alternatively, $100 \mathrm{cc}$ could have been chosen instead of the $10 \%$ volume, but the dose to the $10 \%$ volume provides much of this same information, and for consistency, we have chosen the $50 \%$ and $10 \%$ volume limits for every critical anatomical structure throughout the body for SBRT. For many critical structures that have highly variable size from one patient to the next, like bladder, heart, and stomach, the adaptive nature of the $10 \%$ volume is particularly useful. Statistical techniques can eventually be used to determine optimal volumes and doses [20-21], but the remarkable convenience of Emami's limits is that the dose limits for all critical structures were assigned common volumes for ease of use. Whenever it is effective, we aim to follow this approach.

The majority of the dose-volume limits in Table 1 are specified at either 1cc or 10cc. Rather than making a difficult choice between the two, we believe that both have importance in clinical decision making and that both should be used in the unified format. The 1cc skin dose limits represent a conveniently small volume of skin that could suffer damage from an SBRT treatment. The dose gradients in SBRT are often steep enough that 1cc of skin receives a high dose, even though the remainder of skin meets all other dose tolerance limits. The 10cc dose limits represent a large volume that could experience an adverse event, so these volumes are generally held to about $15 \%$ lower dose than the 1cc volumes are allowed to receive. Maximum dose tolerance limits are less accurate, especially for moving anatomy, and their relevance has been questioned, but we prefer to keep them as part of the unified dose tolerance limit format until long term statistical outcomes analysis has proven or disproven their predictive value. Obviously these are just logical and geometrical arguments, but the ongoing clinical trials are demonstrating the effectiveness of this rationale. Statistical analysis of long-term adverse event data will ultimately determine the ideal volumes and doses, but these limits have been clinically useful in the meantime.

When the SBRT skin dose tolerance limits from Table 1 are plotted as in Fig. 2, a natural partitioning of the low-risk and high-risk limits may be seen. The dashed lines illuminate a trend among the 10cc and max dose limits of Timmerman, RTOG 0618, and RTOG 0813, for one, three, and five fractions. However, none of them have provided a 1cc limit, so we have interpolated between these and the maximum dose limits with a weighting according to an 
average characteristic of our DVH data. Since these dashed lines are mostly 5 Gy or more below other reputable clinical trials, we have designated these as the low-risk dose tolerance limits. In the tabular portion of Fig. 2, the selected published dose tolerance limits are highlighted in boldface, and the linearly interpolated or extrapolated values are italicized.

For the high-risk limits, the adverse events reported at 48 Gy and 54 Gy max dose in three fractions demarcate a level to avoid until it is better understood. For the four-fraction 10cc limit, we selected the RTOG 0915 limit of 33.2 Gy instead of the Accuray STARS limit of 35 Gy because of three adverse events reported above $35 \mathrm{~Gy}$ [9], although as more data becomes available it may be shown that either level is appropriate for high risk cases. The Accuray STARS protocol did not provide max dose limits, and in light of the adverse events reported at $48 \mathrm{~Gy}$ and $54 \mathrm{~Gy}$, to be conservative we incremented the unified dose tolerance limits to be just $1 \mathrm{~Gy}$ higher than the 1cc limits. Similarly, to extrapolate to five-fraction limits, we only added 1 Gy to the four-fraction 10cc, 1cc, and max limits. We expect that these preliminary high-risk dose tolerance limits are below the TD 50/5 limits we are seeking, but until sufficient data is analyzed caution is advised.

Monte Carlo calculations of the actual doses given to the 42 patients are shown in Fig. 2 as the solid dots. The doses of the three patients that experienced CTCAE v3 Grade 1 mild erythema are marked with a square around the corresponding dot. It may be seen that all three of the patients exceeded the low-risk 1cc and max dose limits, and one of them exceeded the 10cc dose limit. All three of these patients were treated with three fractions and in two to three elapsed days. The Grade 1 symptoms for all three patients were observed at the initial onemonth follow-up visit and all had resolved by the three month follow-up. Overall, the 42 patients exceeded low-risk limits 18 times with no Grade 2 or higher adverse events, thus helping to validate the safety of the low-risk limits. Whenever feasible we still try to meet the low-risk dose tolerance limits, but in many cases they would prevent the optimal dose from being delivered to the tumor.

The DVHs for one of the three patients that experienced Grade 1 erythema are shown in Fig. 1, evaluated according to RTOG 0618. It may be seen from the figure that the dose received by $95 \%$ of the PTV (D95) is 6110.8 cGy, meeting the RTOG prescription dose requirement. However, the 24 Gy max skin dose limit is exceeded by $9.6 \mathrm{~Gy}$, and if the plan had been scaled down to meet this dose tolerance limit, the tumor could only receive $43.7 \mathrm{~Gy}$, far less than the desired $60 \mathrm{~Gy}$. The same treatment plan is evaluated with respect to the unified low-risk and high-risk skin dose tolerance limits in Fig. 3. Although three of the low-risk limits are exceeded (Fig. 3A), all of the high-risk limits are met (Fig. $3 B$ ), allowing the patient to receive the desired dose to the tumor. The unified dose tolerance limits provide more flexibility, but each physician must still carefully assess which dose limits are appropriate for each of their patients, and patients should be explicitly consented whenever their treatment plan approaches any of the high risk dose tolerance limits. 

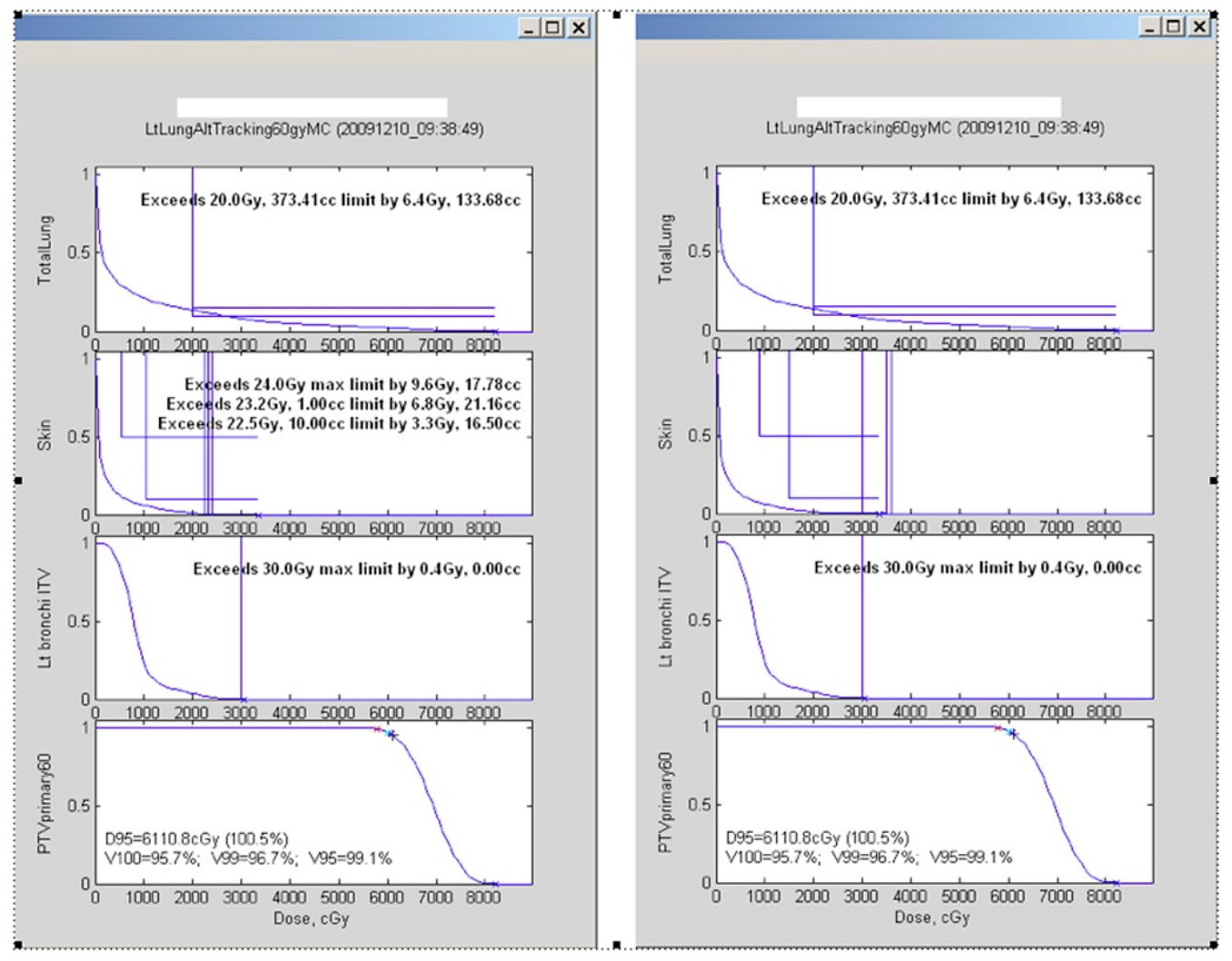

FIGURE 3: The sample patient from Fig. 1 evaluated against A) the unified low-risk skin dose tolerance limits, and B) the unified high-risk skin dose tolerance limits

The sample patient from Fig. 1 evaluated against A) the unified low-risk skin dose tolerance limits, and B) the unified high-risk skin dose tolerance limits.

\section{Discussion}

The unified SBRT skin dose tolerance limits in Fig. 2 provide information for all fractionations from one to five. The limits have been partitioned into high-risk and low-risk categories from which physicians can decide appropriate risk for the stage and condition of each patient. The same set of volumes is utilized consistently regardless of risk or fraction number. In contrast, the current state of the literature as presented in Table 1 is still inconsistent and lacking many important limits such as A) two-fraction limits, B) low-risk 1cc dose limits, and C) high-risk max dose limits. Although the statistically estimated dose limits have not yet been determined, this unified framework and these preliminary dose limits are useful clinically and can guide future research.

The definitions for skin in Table 1 vary from $1 \mathrm{~mm}$ thick to $10 \mathrm{~mm}$ thick. The RTOG protocols [11-14] and the Accuray STARS protocol [15] specify skin as the outer 5mm of the body surface, which is an advantageous definition for several reasons. The thickness of human skin varies throughout the body and typically ranges from $1.5-5 \mathrm{~mm}$ [22]. Due to the skin-sparing effect of high energy photons, the dose gradient at the patient's surface is much steeper than at depth $5 \mathrm{~mm}$, so the $5 \mathrm{~mm}$ thick contours have the additional advantage of improved dose calculation accuracy. Although the shallower dose gradient at depth $5 \mathrm{~mm}$ alleviates the effects of contouring errors, it is still of utmost importance to meticulously check the skin autocontours 
and correct where necessary. For $6 \mathrm{MV}$ photons the depth of maximum dose of a radiation beam is $15 \mathrm{~mm}$, and the depth of maximum dose increases at higher energies. Therefore, for photon energies of $6 \mathrm{MV}$ and greater, the dose at $5 \mathrm{~mm}$ will be higher than at shallower depths, so the $5 \mathrm{~mm}$ thick skin contour serves as a convenient and consistent upper bound of actual skin dose - in all of our cases, the maximum dose within the skin contours has occurred at a distance $5 \mathrm{~mm}$ from the surface. For these reasons, we recommend the $5 \mathrm{~mm}$ thick skin contours, and we have used this definition for all the unified dose tolerance limits in Fig. 2.

Skin contours should also include any skin folds, which are frequently not automatically included by autocontouring algorithms. Skin folds may appear to be more sensitive to radiation because they do not benefit from the skin sparing effects, since they are often completely surrounded by other tissue. The cut-off superior and inferior slabs of the CT should not be contoured as skin unless skin is actually present in these locations, as in the scalp on the top of the head [11]. Treatment plans with more than five beams can spread the entrance and exit doses more uniformly to help reduce skin toxicity.

One of the adverse events shown in Table 1 was reported at a depth of $1 \mathrm{~mm}$ [7]. This dose of 30 Gy in three fractions is substantially lower than our interpolated high-risk limit of $36 \mathrm{~Gy}$. However, if the skin sparing effects of high energy photons are taken into account, the dose at depth $5 \mathrm{~mm}$ would have been 25 - 50\% higher, which would have exceeded our unified high-risk limit. In any case, a percentage of patients is expected to experience Grade 3 adverse events at doses near the high-risk limits; the physician must decide the best risk-benefit ratio for each patient. This presented set of unified high-risk limits is expected to be lower than the $50 \%$ risk level, and might even be lower than the $5 \%$ risk level. The ongoing clinical trials will statistically determine their associated risk level, but in the meantime, great caution should be exercised. The actual 50\% risk dose levels are usually extrapolated using statistical analysis because it is uncommon for such high risk to be required for a patient's evaluation.

Of all 42 CUH cases, the lowest ratio of lung tumor prescription dose to maximum skin dose was $5 / 4$. Using this as an approximate lower bound, and based on the high-risk max dose limits, the achievable prescriptions to lung tumors should usually be at least 45Gy in three fractions, 51Gy in four fractions, and 52Gy in five fractions. This means that although skin dose could commonly be a limiting factor in meeting the RTOG 0618 prescription requirement of 60 Gy in three fractions, it should not often preclude the RTOG 0915 prescription of $48 \mathrm{~Gy}$ in four fractions or the RTOG 0813 prescription of $50 \mathrm{~Gy}$ in five fractions. Therefore, even though the maximum tolerated skin dose for SBRT most likely has not yet been reached, these unified dose tolerance limits provide a very useful range for clinical applications.

\section{Conclusions}

SBRT dose tolerance limits have progressed beyond the pure research phase and there now exist numerous publications and clinical trials. A few papers have presented statistical analysis of dose limits for select critical structures, but sufficient long term data is generally lacking. SBRT data is still in the era of expert opinion. It is an ideal time to consolidate the numerous published limits to establish consensus of expert opinion, for current clinical practice and to help focus the forthcoming statistical results into the most clinically useful format. It is our hope that the unified framework presented in this manuscript will facilitate this mission.

\section{Additional Information}

\section{Disclosures}

Human subjects: Consent was obtained by all participants in this study. The Cooper University Hospital Institutional Review Board issued approval \# 10-094EX. Animal subjects: All authors 
have confirmed that this study did not involve animal subjects or tissue. Conflicts of interest: In compliance with the ICMJE uniform disclosure form, all authors declare the following:

Payment/services info: All authors have declared that no financial support was received from any organization for the submitted work. Financial relationships: All authors have declared that they have no financial relationships at present or within the previous three years with any organizations that might have an interest in the submitted work. Other relationships: The first author developed the DVH Evaluator software tool which is an FDA-cleared product in commercial use. None of the authors has received any funding for this research.

\section{References}

1. Timmerman R, Paulus R, Galvin J, Michalski J, Straube W, Bradley J, Fakiris A, Bezjak A, Videtic G, Johnstone D, Fowler J, Gore E, Choy H: Stereotactic body radiation therapy for inoperable early stage lung cancer. JAMA. 2010, 303:1070-6.

2. Timmerman R, McGarry R, Yiannoutsos C, Papiez L, Tudor K, DeLuca J, Ewing M, Abdulrahman R, DesRosiers C, Williams M, Fletcher J: Excessive toxicity when treating central tumors in a phase II study of stereotactic body radiation therapy for medically inoperable early-stage lung cancer. J Clin Oncol. 2006, 24:4833-9.

3. Song SY, Choi W, Shin SS, Lee SW, Ahn SD, Kim JH, Je HU, Park CI, Lee JS, Choi EK: Fractionated stereotactic body radiation therapy for medically inoperable stage I lung cancer adjacent to central large bronchus. Lung Cancer. 2009, 66:89-93.

4. Emami B, Lyman J, Brown A, Coia L, Goitein M, Munzenrider JE, Shank B, Solin LJ, Wesson M: Tolerance of normal tissue to therapeutic radiation. Int J Radiat Oncol Biol Phys. 1991, 21:109-122.

5. Quantitative Analyses of Normal Tissue Effects in the Clinic (QUANTEC): an introduction to the scientific issues. 2010, 76:3-9. 10.1016/j.ijrobp.2009.09.040

6. Timmerman RD: An overview of hypofractionation and introduction to this issue of seminars in radiation oncology. Semin Radiat Oncol. 2008, 18:215-222.

7. Rusthoven KE, Kavanagh BD, Burri SH, Chen C, Cardenes H, Chidel MA, Pugh TJ, Kane M, Gaspar LE, Schefter TE: Multi-institutional phase I/II trial of stereotactic body radiation therapy for lung metastases. J Clin Oncol. 2009, 27:1579-84.

8. Koto M, Takai Y, Ogawa Y, Matsushita H, Takeda K, Takahashi C, Britton KR, Jingu K, Takai K, Mitsuya M, Nemoto K, Yamada S: A phase II study on stereotactic body radiotherapy for stage I non-small cell lung cancer. Radiother Oncol. 2007, 85:429-34.

9. Chang J, Balter P, Dong L, et al.: Stereotactic body radiation therapy in centrally and superiorly located stage I or isolated recurrent non-small-cell lung cancer. Int J Radiat Oncol Biol Phys. 2008, 72:967-971.

10. Welsh J, Thomas J, Shah D, Allen PK, Wei X, Mitchell K, Gao S, Balter P, Komaki R, Chang JY: Obesity Increases the Risk of Chest Wall Pain from Thoracic Stereotactic Body Radiation Therapy. Int J Radiat Oncol Biol Phys. 2011, 81:91-6. 10.1016/j.ijrobp.2010.04.022

11. A phase II trial of stereotactic body radiation therapy (SBRT) in the treatment of patients with operable stage I/II non-small cell lung cancer. Radiation Therapy Oncology Group 0618. Accessed: 2011: http://www.rtog.org/ClinicalTrials/ProtocolTable.aspx.

12. Phase II/III study of image-guided radiosurgery/SBRT for localized spine metastasis. Radiation Therapy Oncology Group 0631. Accessed: 2011: http://www.rtog.org/ClinicalTrials/ProtocolTable.aspx.

13. Seamless phase I/II study of stereotactic lung radiotherapy (SBRT) for early stage, centrally located, non-small cell lung cancer (NSCLC) in medically inoperable patients. Accessed: 2011: http://www.rtog.org/ClinicalTrials/ProtocolTable.aspx.

14. A randomized phase II study comparing 2 stereotactic body radiation therapy (SBRT) schedules for medically inoperable patients with stage I peripheral non-small cell lung cancer. Accessed: 2011: http://www.rtog.org/ClinicalTrials/ProtocolTable.aspx.

15. International randomized study to compare CyberKnife ${ }^{\circledR}$ stereotactic radiotherapy with surgical resection in stage I non-small cell lung cancer (STARS). Accessed: 2011: http://clinicaltrials.gov.

16. Hoppe BS, Laser B, Kowalski AV, Fontenla SC, Pena-Greenberg E, Yorke ED, Lovelock DM, Hunt MA, Rosenzweig KE: Acute skin toxicity following stereotactic body radiation therapy 


\section{Cureus}

for stage I non-small-cell lung cancer: who's at risk?. Int J Radiat Oncol Biol Phys. 2008, 72:1283-6. 10.1016/j.ijrobp.2008.08.036

17. Kavanagh BD, Schefter TE, Cardenes HR, Stieber VW, Raben D, Timmerman RD, McCarter MD, Burri S, Nedzi LA, Sawyer TE, Gaspar LE: Interim analysis of a prospective phase I/II trial of SBRT for liver metastases. Acta Oncol. 2006, 45:848-55.

18. National Cancer Institute. Common Terminology Criteria for Adverse Events, version 3.0 . (2006). Accessed: 2011: http://ctep.cancer.gov/forms/CTCAEv3.pdf.

19. Grimm J, LaCouture TA, Croce RJ, Inhwan Y, Zhu Y, Xue J: Dose tolerance limits and dose volume histogram evaluation for stereotactic body radiotherapy. J Appl Clin Med Phys. 2011, 12:267-92.

20. Dunlap NE, Cai J, Biedermann GB, Yang W, Benedict SH, Sheng K, Schefter TE, Kavanagh BD, Larner JM: Chest Wall Volume Receiving >30 Gy Predicts Risk of Severe Pain and/or Rib Fracture After Lung Stereotactic Body Radiotherapy. Int J Radiat Oncol Biol Phys. 2010, 76:796-801. 10.1016/j.ijrobp.2009.02.027

21. Pettersson N, Nyman J, Johansson KA: Radiation-induced rib fractures after hypofractionated stereotactic body radiation therapy of non-small cell lung cancer: a dose- and volumeresponse analysis. Radiother Oncol. 2009, 91:360-8. 10.1016/j.radonc.2009.03.022

22. Fornage BD, Deshayes JL: Ultrasound of normal skin. J Clin Ultrasound. 1986, 14:619-22. 